\title{
An lncRNA-miRNA-mRNA-ceRNA network regulates intervertebral disc degeneration: A bioinformatics study based on the dataset analysis
}

\author{
Xutao Fan ${ }^{1}$, Guowu Chen ${ }^{1}$, Fengyu $\mathrm{Ma}^{2}$, Bao Qi ${ }^{1}$, Yanhu Liang ${ }^{3}$, Pihao Gong ${ }^{3}$ and Chunyang \\ Meng ${ }^{1,4}$ \\ ${ }^{1}$ Department of Spine Surgery, Affiliated Hospital of Jining Medical University, Jining, Shandong, P.R. China \\ ${ }^{2}$ Department of Spine Surgery, People's Hospital of Rizhao, Shandong, P.R.China \\ ${ }^{3}$ Graduate School of Jining Medical University, Jining, Shandong, P.R.China \\ ${ }^{4}$ Neuropathic Pain Institute for Spinal Nerve of Jining Medical University, Jining, Shandong, P.R.China
}

\begin{abstract}
Intervertebral disc degeneration (IDD) leads to low back pain (LBP). This study aimed to determine the regulation of IDD by competing endogenous RNAs (ceRNAs). We obtained the GSE63492, GSE124272, and GSE129789 datasets from the Gene Expression Omnibus database. The changes of long non-coding RNAs (lncRNAs), microRNAs (miRNAs), and mRNAs in IDD were characterized. The significantly changed mRNAs were subjected to protein-protein interaction analysis using the STRING database, and its functions and involved pathways were analyzed using the DAVID database and gene set enrichment analysis (GSEA). The significant changed lncRNAs, miRNAs and mRNAs were linked in a ceRNA network based on their interactions - predicted by Starbase and miRWalk. Differentially methylated loci of significantly changed mRNAs in early and advanced IDD were compared using the GSE129789 dataset. We identified 245 significantly changed mRNAs, 133 lncRNAs, and 228 miRNAs between patients with IDD and normal individuals. GSEA suggested that 17 pathways related to cell proliferation were activated while 35 cell signaling and immune-related pathways were suppressed in IDD. The following ceRNA network in IDD was built: LINC00665/hsa-miR-7-5p/FZD3, ZNF549; LINC00665/hsa-let-7e-5p/FZD3, ACVR2B; TRG-AS1/hsa-miR-574-5p/ACVR2B, P3H2; TRG-AS1/ hsa-let-7e-5p/FZD3, ACVR2B; and ZNF571-AS1/let-7e-5p/ACVR2B, FZD3. A IncRNA-miRNAmRNA ceRNA network which might regulate the progression of IDD was developed.
\end{abstract}

Key words: Intervertebral disc degeneration - Long non-coding RNA - Differential methylation - Competing endogenous RNA network

\begin{abstract}
Abbreviations: AS_IDD, advanced IDD; BP, biological process; CC, cellular component; ceRNAs, competing endogenous RNAs; DEGs, differentially expressed genes; DEmiRNAs, differentially expressed miRNAs; DEmRNAs, differentially expressed mRNAs; DML, differentially methylated loci; ECM, extracellular matrix; ES_IDD, early stage IDD; FC, fold change; FZD3, Frizzled 3; GEO, Gene expression omnibus; GO, Gene Ontology; IDD, intervertebral disc degeneration; IVD, intervertebral discs; LBP, low back pain; lncRNAs, long non-coding RNAs; MAPK, mitogen-activated protein kinase; miRNAs, microRNAs; NP, nucleus pulposus; $\mathrm{P} 3 \mathrm{H} 2$, prolyl 3-hydroxylase 2; PPI, proteinprotein interaction; TGF, transforming growth factor; TNF, tumor necrosis factor; TRG-AS1, T cell receptor gamma locus antisense RNA 1.
\end{abstract}

Correspondence to: Chunyang Meng, Department of Spine Surgery, Affiliated Hospital of Jining Medical University, 89 Guhuai Road, Jining, Shandong 272029, P.R.China; Neuropathic Pain Institute for Spinal Nerve of Jining Medical University, 133 Hehua Road, Jining, Shandong 272067, P.R.China

E-mail: chunyangmeng16@126.com 


\section{Introduction}

Low back pain (LBP) is a chronic prevalent medical problem that affects over $80 \%$ of adults worldwide and thus has a significant economic impact (Andersson 1999). Intervertebral disc degeneration (IDD) is closely associated with LBP. It is characterized by the progressive structural failure and aging of affected discs (Luoma et al. 2000). Intervertebral discs (IVD) physiologically comprise annulus fibrosus (AF), cartilaginous vertebral endplates (EP), and nucleus pulposus (NP). Multi-factorial risks attributed to the pathogenesis of IDD include genetic predisposition, aging, and lifestyle (smoking and occupation) (Akeda et al. 2015). However, the exact mechanisms involved in IDD remain to be elusive. NP cells function in maintaining IVD integrity through extracellular matrix (ECM) components, such as type II collagen, aggrecan, and type X collagen (Loreto et al. 2011). Accumulating evidence has suggested that NP cell deregulation functions in ECM production, cytokine secretion, altered cell proliferation, and apoptosis, all of which play key roles in IDD pathogenesis ( $\mathrm{Li}$ et al. 2018).

Long noncoding RNAs (lncRNAs) and microRNAs (miRNAs) play regulatory roles in various biological processes (Yao et al. 2019). With a length over 200 nucleotides, lncRNAs deregulate inflammatory and infectious diseases. lncRNAs also play pivotal roles in proliferation, differentiation, migration, invasion, and apoptosis (Zhao $\mathrm{H}$ et al. 2016; Li et al. 2017; Yang et al. 2018). miRNAs comprising 20-22 nucleotides function as regulatory factors in cell proliferation, apoptosis, ECM anabolism, and cytokine release (Liu et al. 2015). IncRNAs may function as competing endogenous RNAs (ceRNAs) in the regulation of target mRNAs by competitively binding with miRNAs. Interactions among lncRNAs, miRNAs, and mRNAs may constitute important mechanisms during IDD pathogenesis (Zhu et al. 2019). A total of 1,854 differentially expressed lncRNAs (DElncRNAs) have been identified in the central NP tissues of patients with IDD. These lncRNAs function in adhesion as well as lysosome and mitogen-activated protein kinase (MAPK) signaling. lncRNA PART1 is thought to activate E2F transcription factor 3, vascular endothelial growth factor, and activing A receptor type 1 (ACVR1) in IDD by sponging miR-34a and miR-148a (Zhao B et al. 2016). Aberrantly increased lncRNA RP11-296A18.3 acts as the ceRNA for miR-138 to depress its target hypoxia-inducible factor 1-alpha, thereby promoting NP cell proliferation and ECM deposition during IDD development (Wang et al. 2017). The expression levels of tumor necrosis factor (TNF), Wnt1, and $\beta$-catenin are enhanced by TUG1 in NP cells, leading to ECM synthesis, apoptosis, and suppression (Chen et al. 2017). lncRNA HCG18 sponges miR-146a-5p, which results in the inhibition of cell proliferation and subsequently promotes NP cell apoptosis (Xi et al. 2017). lncRNA H19 directly sponges miR-22 in clinical IDD specimens and alleviates the inhibitory effects of lymphoid enhancer-binding factor 1 , thus inhibiting NP cell proliferation (Wang et al. 2018). These findings indicate that an lncRNA-miRNA-mRNA axis may have regulatory effects on the mechanism of IDD that could lead to improvement.

DNA methylation is the process of adding methyl groups to cytosine bases in nucleotides that contain $\mathrm{CpG}$, thus forming 5-methylcytosine without changing the DNA sequence. Methylation is an essential mechanism in cancer and neurodegenerative diseases. Hypermethylated caspase recruitment domain-containing protein 14 (CARD14), EF-hand domain family member D2 (EFHD2), and rhotekin 2 (RTKN2) genes are involved in NF- $\kappa \mathrm{B}$ pathway regulation in advanced IDD (AS_IDD) (Myouzen et al. 2012). In addition, methylation in the transforming growth factor (TGF)- $\beta$ and Hedgehog (Hh) signaling pathways is involved in cell adhesion and the cell matrix in IDD (Pusapati et al. 2014).

This study aimed to determine whether an lncRNA-miRNA-mRNA ceRNA network plays a regulatory role in IDD, elucidate the pathological mechanism of IVD degeneration, and uncover targets for novel IDD therapeutic strategies.

\section{Materials and Methods}

\section{Gene expression omnibus (GEO) datasets}

The datasets of GSE63492, GSE 124272 and GSE129789 were downloaded from GEO database (https://www.ncbi. nlm.nih.gov/geo/) for further analysis. The non-coding RNA dataset GSE63492 was deposited by Wang et al. (2011), which included data from 5 IDD samples (grade IV-V, average age: $36.80 \pm 7.33$ years old) and 5 control samples (grade I, average age: $40.80 \pm 7.50$ years old). The expression profile GSE124272 was deposited by Wang et al. (2019), which contained data from 8 IDD patients aged between 33 and 60 years, and 8 volunteers aged between 19 and 23 years. The DNA methylation data profile GSE129789 was deposited by Ikuno et al. (2019) which included data from 8 early stage (ES_IDD, grade I-III, average age: $45.58 \pm 15.70$ years old) and 8 AS_IDD (grade IV, average age: $65.38 \pm 10.53$ years old) samples. In these datasets, "protein_coding" was defined as mRNA while "antisense," "sense_intronic," "lincRNA," "sense_overlapping," "processed_transcripts," “ 3 ' overlapping_ncRNA," and "non_coding" were defined as ncRNA using the annotation software gtf (Release 26, GRCh38.p10).

\section{Identification of differentially expressed genes (DEGs)}

DEGs of GSE63492 and GSE124272 were analyzed using the limma package (version 3.10.3) in R (The R Foundation, Vienna, Austria). Statistical tests were applied to filter 
out DEGs in the dataset according to $p$ values using the Benjamini-Hochberg method. $\mid$ Log fold change (FC) $\mid \geq 1$, and $p \leq 0.05$ were used to identify differentially expressed mRNAs (DEmRNAs) and differentially expressed miRNAs (DEmiRNAs). $|\operatorname{LogFC}| \geq 0.5$ and $p \leq 0.05$ were used to identify DElncRNAs.

\section{Protein-protein interaction (PPI) network}

A PPI network was constructed based on the STRING database with DEmRNAs as the input set. Topological features, including edges and nodes, were calculated to identify crucial genes.

\section{Functional enrichment and pathway analyses}

The Gene Ontology (GO) of DERNAs was determined using the DAVID database version 6.7 (https://david-d.ncifcrf. gov/) and visualized using the GOplot package in R. Biological process (BP), cellular component (CC), and molecular function (MF) terms were annotated.

Kyoto Encyclopedia of Genes and Genomes (KEGG) pathway enrichment of DERNAs was implemented using Gene Set Enrichment Analysis version 3.0 (GSEA; https:// www.genome.jp/kegg/) with adjusted $p<0.05$.

\section{Construction of the mRNA-lncRNA co-expression network}

Pearson correlation coefficients of DElncRNAs and DEmRNAs were calculated using the psych package in $\mathrm{R}$ with the corr.test method ( $\mathrm{ci}=\mathrm{F}$, adjust $=$ "BH") and visualized using Cytoscape version 3.7.0 software (www.cytoscape.org) with adjusted $p<0.05$ and $r \geq 0.8$.

\section{Construction of miRNA regulatory and ceRNA networks}

The DEmiRNAs that target DElncRNAs in the lncRNAmRNA co-expression network were predicted using starBase version 3.0 (http://starbase.sysu.edu.cn/). The DEmiRNAs that target DEmRNAs in the IncRNA-mRNA co-expression network were predicted using miRWalk (version 3.0) combined with miRDB (http://www.mirdb.org/), miRTarBase (http://mirtarbase.mbc.nctu.edu.tw/), and TargetScan (http://www.targetscan.org/vert_71/). A ceRNA network containing DEmRNAs, DElncRNAs, and DEmiRNAs was constructed by integrating lncRNA-mRNA, lncRNAmiRNA, and miRNA-mRNA interactions using Cytoscape version 3.6.1 software.

\section{Differentially methylated loci identification}

Genome-wide differentially methylated loci (DML) between ES_IDD and AS_IDD in the GSE 129789 dataset were com- pared using the limma package with $p \leq 0.05$ and $|\log \mathrm{FC}| \geq 1$. The DML and DEmRNAs were compared to obtain the methylated loci in DEmRNAs.

\section{Results}

\section{Differential expression analysis}

Here, we analyzed 18,295 mRNAs, 2,128 lncRNAs, and 368 miRNAs. Based on the thresholds of $p<0.05$ and $|\operatorname{logFC}| \geq 1$ for DEmRNAs and DEmiRNAs as well as $p<0.05$ and $|\log \mathrm{FC}| \geq 0.5$ for DElncRNAs, we identified 245 DEmRNAs, of which 119 were upregulated and 126 were downregulated. We also identified 133 DElncRNAs, among which 63 were upregulated and 70 were downregulated, and 228 DEmiRNAs that included 138 upregulated and 90 downregulated miRNAs between patients with IDD and the controls (Fig. $1 \mathrm{~A}, \mathrm{C}, \mathrm{E})$. Hierarchical cluster heat maps indicated the top 10 upregulated and downregulated genes that distinguished IDD from the controls (Fig. 1B,D,F).

\section{PPI network construction}

A PPI network was constructed from the filtered DEmRNAs using the STRING database. This network contained 163 nodes and 514 edges with an average connection degree of 4.27, an average local clustering coefficient of 0.425 , and a PPI enrichment $p<1 \mathrm{E}-16$. Budding uninhibited benzimidazoles 1 (BUB1), hyaluronan mediated motility receptor (HMMR), histone $\mathrm{H} 2 \mathrm{~B}$ type 2-E (HIST2H2BE), arginase 1 (ARG1), and cathepsin G (CTSG) were considered as hub genes because of their high degree in the PPI network (Fig. 2).

\section{GO enrichment analysis}

Five BP terms and nine CC terms for the 163 nodes in the PPI network were significantly enriched (Fig. 3A). The BPs that were regulated during IDD progression and enriched in the analysis included "cell division," "chromosome segregation," "defense response to bacterium," "mitotic nuclear division," "response to yeast." Furthermore, "extracellular exosome," "specific granule," "extracellular space," "secretory granule," "plasma membrane," "condensed chromosome kinetochore," "cell surface," "extracellular region," and "neuronal cell body" were enriched in the CC function (Fig. 3B). These findings indicated that IDD is regulated through various pathways.

\section{KEGG pathway analysis}

Next, KEGG pathways enriched by the nodes in the PPI network were analyzed using GSEA. According to the threshold 
of adjusted $p<0.05,52$ pathways were significantly enriched, including 17 activated pathways (normalized enrichment score $(\mathrm{NES})>0$ ) and 35 inhibited pathways $(\mathrm{NES}<0)$. Pathways that were associated with cell proliferation included "ribosome," "DNA replication," "aminoacyl tRNA biosynthesis," "cell cycle," "nucleotide excision repair," "ribosome biogenesis in eukaryotes," and "RNA polymerase." "Complement and coagulation cascades," "necroptosis," "pertussis," "toll like receptor signaling pathway," "leishmaniasis," "fc $\gamma$-mediated phagocytosis", and "complement and coagulation cascades" related to cell-cell interaction and immunity were suppressed (Fig. 4).
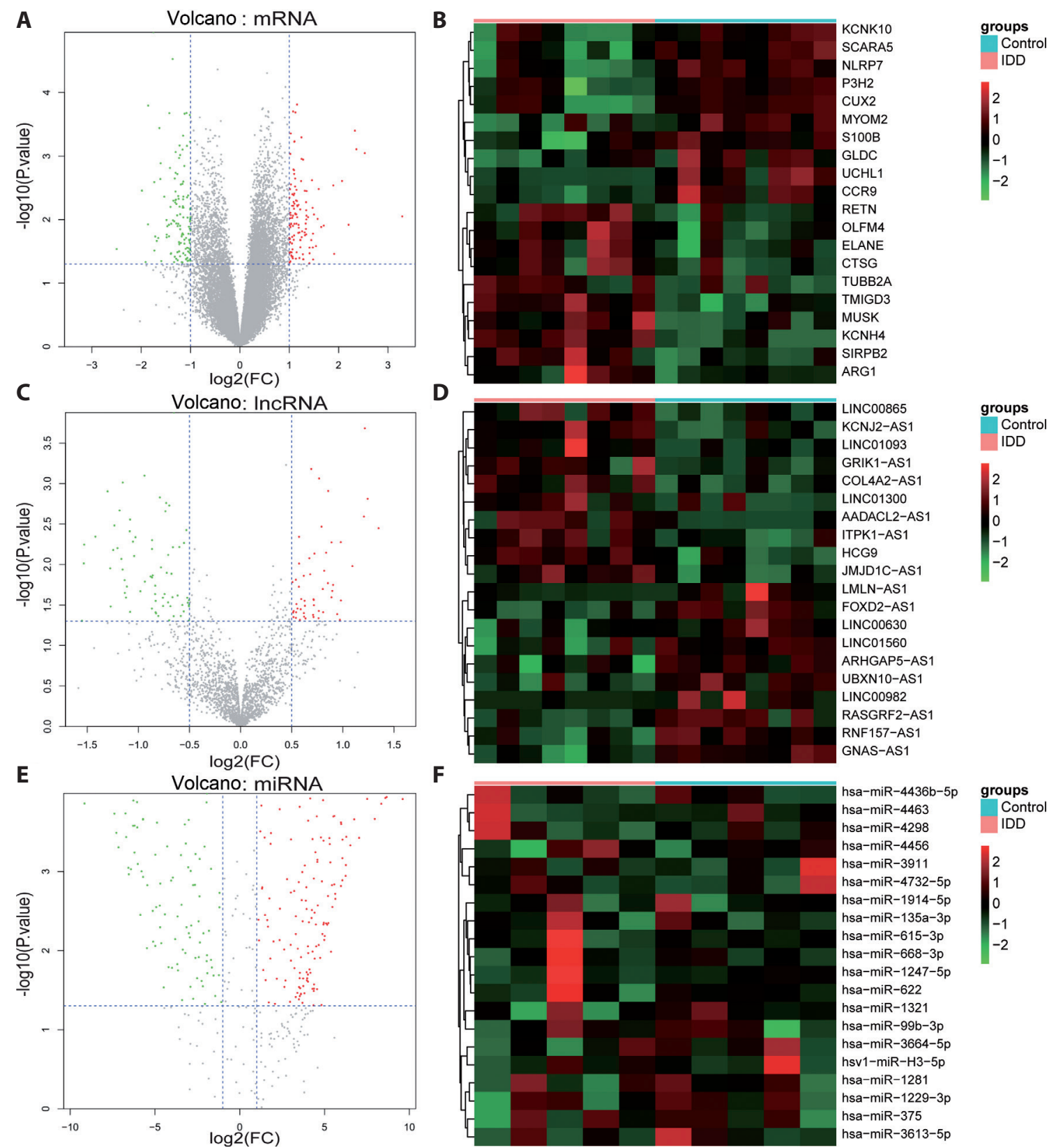

Figure 1. Differentially expressed genes (DEGs), lncRNAs (DElncRNAs), and miRNAs (DEmiRNAs) between the IDD and normal samples. Left: Volcano plots of DEGs (A), DElncRNAs (C), and DEmiRNAs (E). Vertical dotted lines, $\mid \log$ fold change (FC) $\mid \geq 1$, and $p \leq 0.05$ for mRNAs and miRNAs as well as $|\log \mathrm{FC}| \geq 0.5$ and $p \leq 0.05$ for $\operatorname{lncRNAs}$. Red and green spots represent upregulated and downregulated genes, respectively, in IDD compared to normal samples. Right: Hierarchical clustering of the top 10 DEGs. The heatmap shows the top 10 upregulated and downregulated DEGs in descending order according to $|\log \mathrm{FC}| \geq 1$ and $p \leq 0.05$ for mRNAs (B) and miRNAs (D) as well as $|\log \mathrm{FC}| \geq 0.5$ and $p \leq 0.05$ for $\operatorname{lncRNAs}(\mathbf{F})$. IDD, intervertebral disc degeneration. 


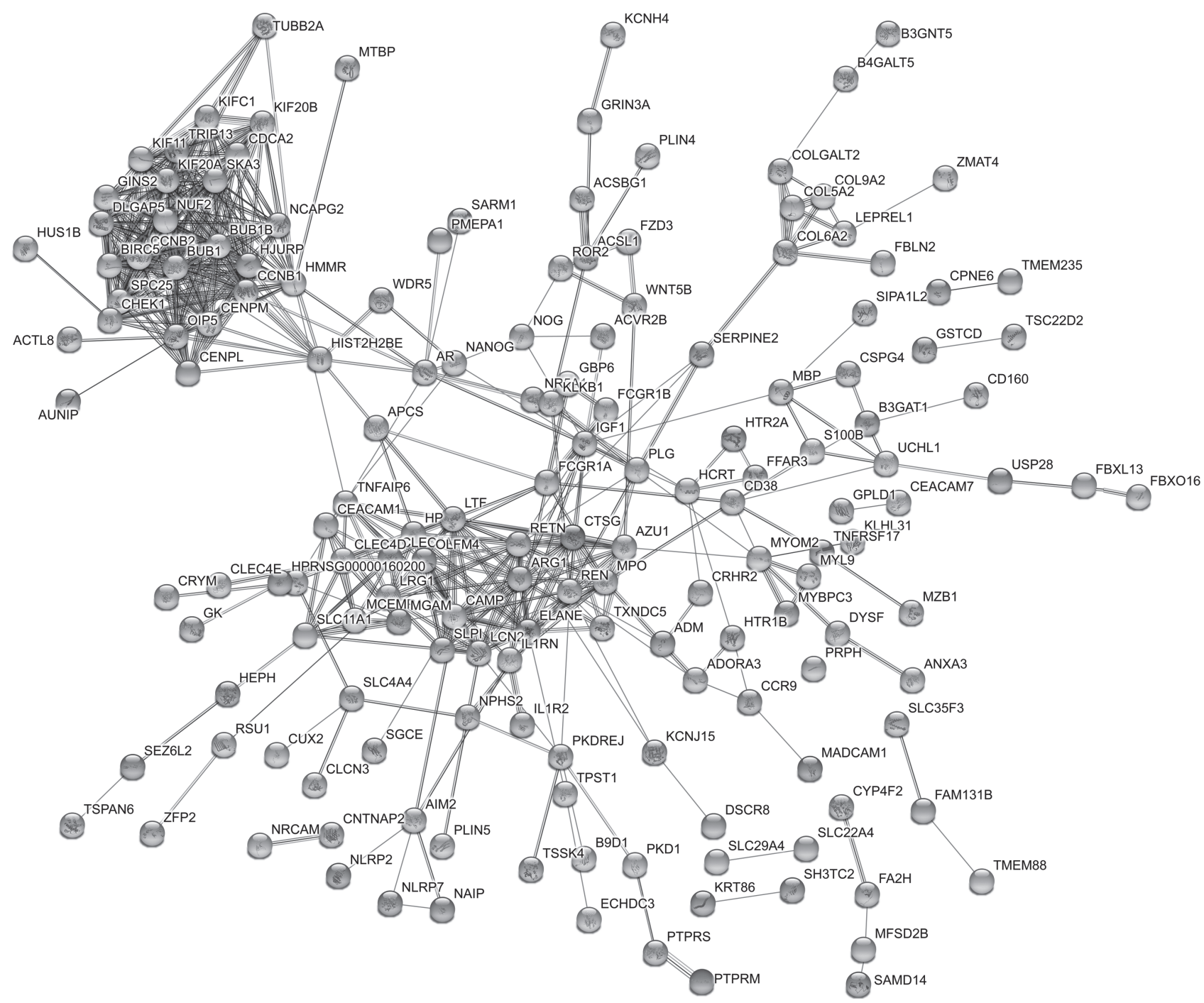

Figure 2. Protein-protein interaction (PPI) network determined using immune-related genes. The nodes represent proteins of DEGs. Larger nodes (proteins) indicate more interactions (higher degree).

\section{Construction of the IncRNA-mRNA co-expression network}

Positively coordinated DEmRNAs and DElncRNAs were screened to construct lncRNA-mRNA network using the parameters $\mathrm{r} \geq 0.8$ and $\mathrm{BH}<0.05$. This network contained 156 nodes that consisted of 93 DEmRNAs, 63 DElncRNAs, and 236 edges. Closely connected $\operatorname{lncRNA}$ and mRNA pairs included JARID2-AS1, MTBP, STARD7-AS1, PMEPA1, SATB1-AS1, and RARA-AS1 (Fig. 5).

\section{Construction of the ceRNA network}

miRNA-mRNA and miRNA-lncRNA networks were further constructed by mapping the lncRNAs and mRNAs into the miRWalk database or starBase, respectively. Then, we integrated these three networks to retain overlapped interactions to construct a ceRNA network. This ceRNA network included four DEmRNAs, three DElncRNAs, and three DEmiRNAs. Notably, upregulated TRG-AS1 may function as a ceRNA to activate ACVR2B and prolyl 3-hydroxylase 2 (P3H2) by inhibiting hsa-miR-574-5p as well as Frizzled 3 (FZD3; a member of the FZD family) and ACVR2B by suppressing hsa-let-7e-5p. ZNF571-AS1 sponged hsa-let-7e-5p, led to the upregulation of ACVR2B and FZD3. Upregulated LINC00665 may similarly suppress the inhibitory effects of hsa-miR-7-5p on FZD3 and ZNF549 and function as the ceRNA of FZD3 and ACVR2B by sponging hsa-let-7e-5p. Our findings from the IncRNA-miRNA-mRNA network indicated that hsa-let-7e-5p directly regulates three lncRNAs and is thus particularly important (Fig. 6). 


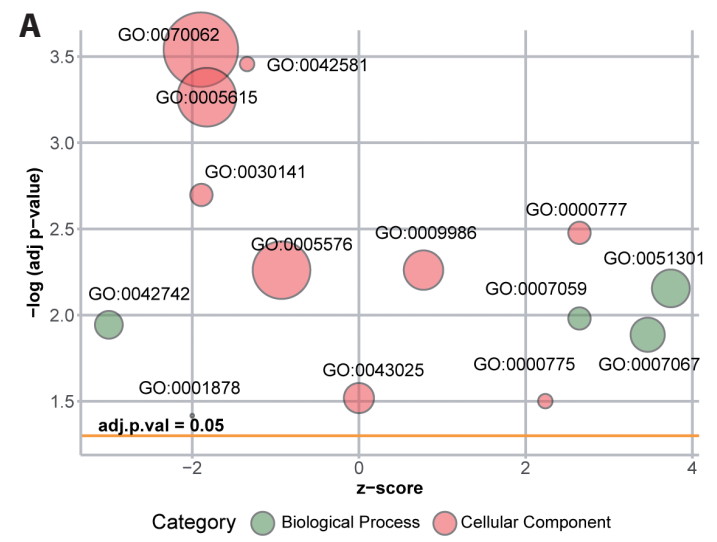

\begin{tabular}{cc} 
ID & Description \\
\hline GO:0051301 & cell division \\
GO:0007059 & chromosome segregation \\
GO:0042742 & defense response to bacterium \\
GO:0007067 & mitotic nuclear division \\
GO:0001878 & response to yeast \\
GO:0070062 & extracellular exosome \\
GO:0042581 & specific granule \\
GO:0005615 & extracellular space \\
GO:0030141 & secretory granule \\
GO:0005886 & plasma membrane \\
GO:0000777 & condensed chromosome kinetochore \\
GO:0009986 & cell surface \\
GO:0005576 & extracellular region \\
GO:0043025 & neuronal cell body \\
GO:0000775 & chromosome, centromeric region \\
\hline
\end{tabular}

\section{GO Terms}

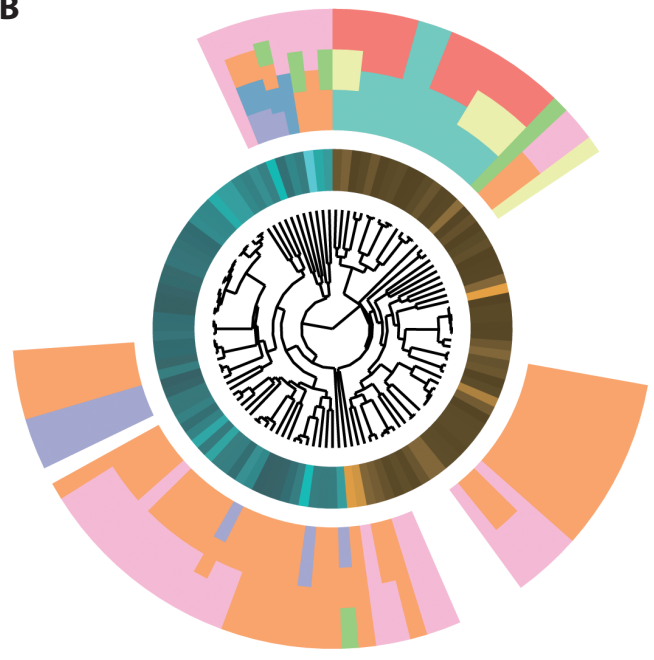

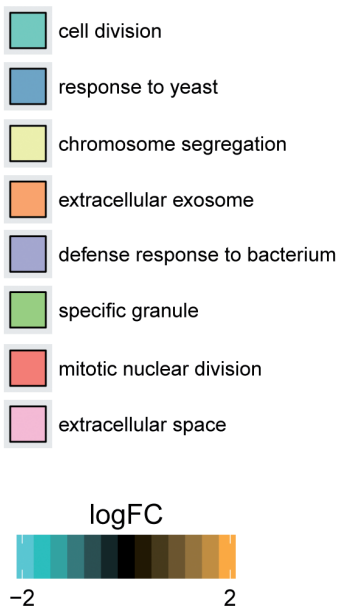

Figure 3. Gene ontology (GO) enrichment analysis. A. Top bubble plot of GO terms. x-axis, z-score; y-axis, negative $\log$-adjusted $p$. Bubble area positively correlates with gene numbers in the indicated term. Green, biological process term; pink, cellular component term. B. GO gene clusters in the top eight GO terms grouped by expression amount. GO, $\log \mathrm{FC}$, and related genes from the outer to inner circles.
A

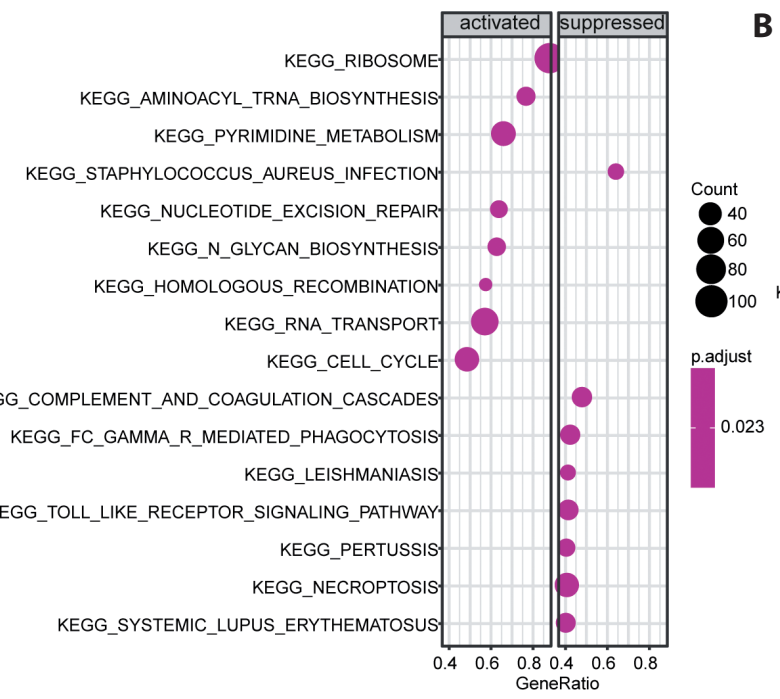

B

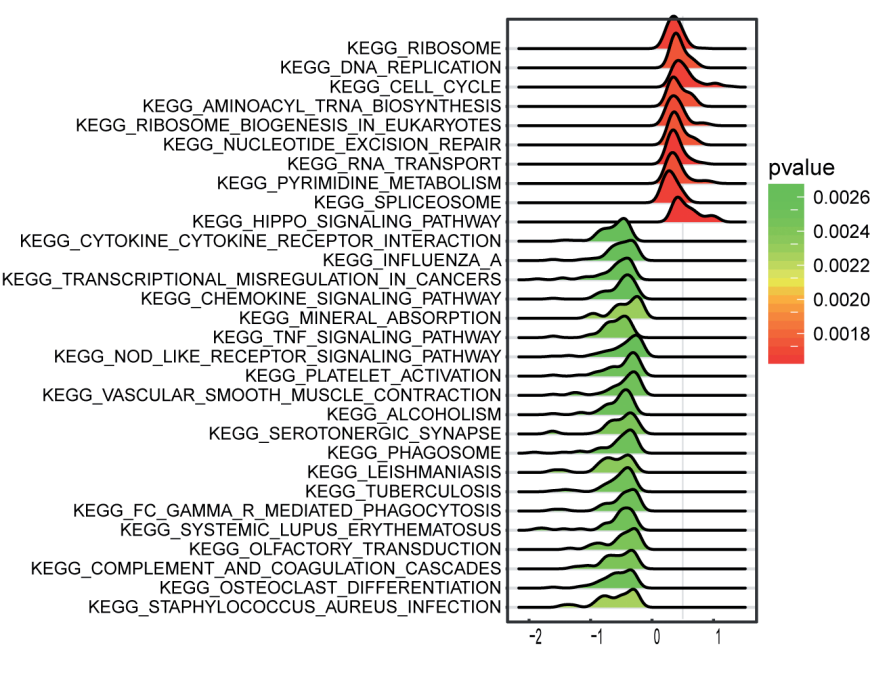

Figure 4. Hierarchical clustering analysis of gene expression and functional analysis. A. Dot plot of the dysregulated pathways in intervertebral disc degeneration (IDD) samples compared to normal samples. Color intensity of the nodes indicates degree of Kyoto Encyclopedia of Genes and Genomes pathway enrichment. Horizontal axis, gene ratio as a proportion of differential genes in entire gene set. Size represents the number of counts in a specific pathway. B. Ridge plot of dysregulated pathways. Joy plot shows the PI3K/ Akt pathway. Color intensity in the peaks indicates enrichment significance. Horizontal axis, $p$ values. 


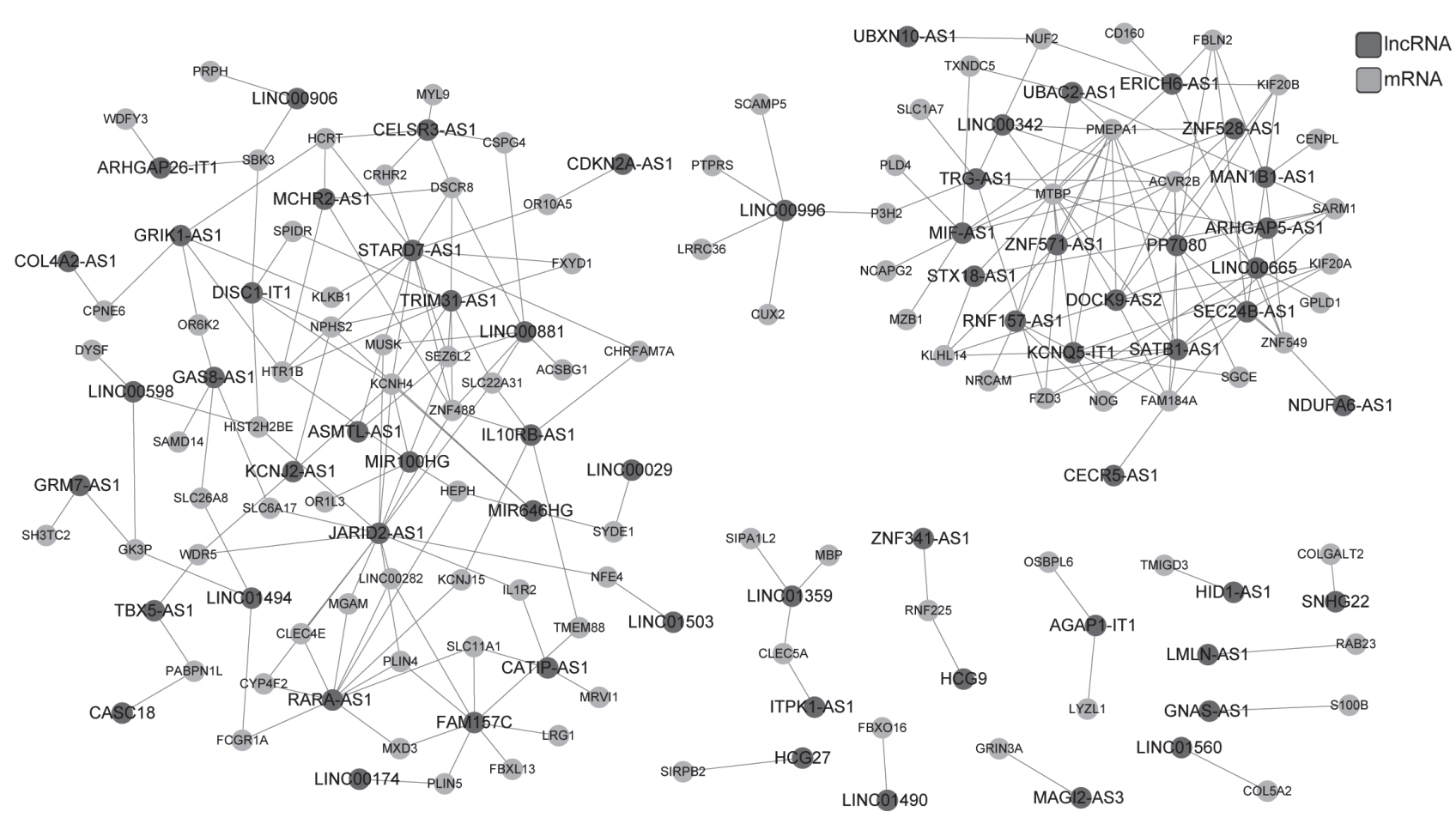

Figure 5. Construction of the lncRNA-mRNA network using DEmRNAs and DElncRNAs. Gene pairs were selected based on a correlation ratio $\geq 0.8$ and adjusted $p<0.05$. Light gray, mRNA; dark gray, lncRNA. Nodes represent proteins while edges represent interactions. lncRNA, long non-coding RNA; DEmRNAs, differentially expressed mRNAs.

\section{Identification of DML in ES_IDD and AS_IDD}

In the GSE129789 dataset, 2,834 methylated loci associated with 208 DEmRNAs were identified. By calculating the statistical significance between the ES_IDD and AS_IDD samples, 161 DMLs were identified according to the thresholds of $p<0.05$ and $|\operatorname{logFC}| \geq 1$, including 48 hypermethylated loci and 113 hypomethylated loci in the AS_IDD samples (Fig. 7A). These DML corresponded to 77 DEmRNAs. Among the hypomethylated DML, the significant loci were enriched in COL5A2, SLC4A4, SLC35F3, IGF1, TSC22D2, BNC2, FAM184A, and SLC6A17 (Fig. 7B). The key hub gene in the PPI network, ZNF549, was differentially methylated at the gene locus cg06458239 in the AD_IDD samples.

\section{Discussion}

In this study, we constructed the following ceRNA network in IDD: LICN00665/hsa-miR-7-5p/FZD3 and ZNF549, LINC00665/hsa-let-7e-5p/FZD3 and ACVR2B, TRG-AS1/ hsa-miR-574-5p/ACVR2B and P3H2, TRG-AS1/ hsa-let7e-5p/FZD3 and ACVR2B, and ZNF571-AS1/hsa-let-7e-5p/ ACVR2B and FZD3. Upregulated LINC00665 may suppress the inhibitory effects of hsa-miR-7-5p on FZD 3 and ZNF549 as well as function as a ceRNA of FZD3 and ACVR2B by sponging hsa-let-7e-5p. TRG-AS1 may function as a ceRNA to inhibit the suppressive effects of hsa-miR-574-5p on ACVR2B and P3H2 as well as hsa-let-7e-5p on FZD3 and ACVR2B. ZNF571-AS1 sponging hsa-let-7e-5p led to the upregulation of ACVR2B and FZD3. Moreover, we identified the aberrant methylation loci in DEmRNAs during IDD progression.

ACVR2B binds kinase and in turn activates downstream molecules, such as $\mathrm{SMAD} 2 / 3$, which regulate cell proliferation or differentiation (Han et al. 2013). The noncanonical ACVR2B signaling pathway also regulates MAPKs and muscle growth as well as blocks myogenic progression (Han et al. 2013). The present study identified a novel function of ACVR2B together with LINC00665 and hsa-let-7e-5p in IDD.

FZD3 is required for axonal development (Hua et al. 2014) and functions in neural crest proliferation (Chai et al. 2015). It is a key factor in sarcoma and breast carcinoma pathogenesis by regulating cell division cycle 25 phosphatase through the noncanonical WNT signaling pathway (Vijayakumar et al. 2011). The present investigation revealed a new role of FZD3 in IDD regulation via the LINC00665/hsa-miR-7-5p/ FZD3 and LINC00665/hsa-let-7e-5p axes. 


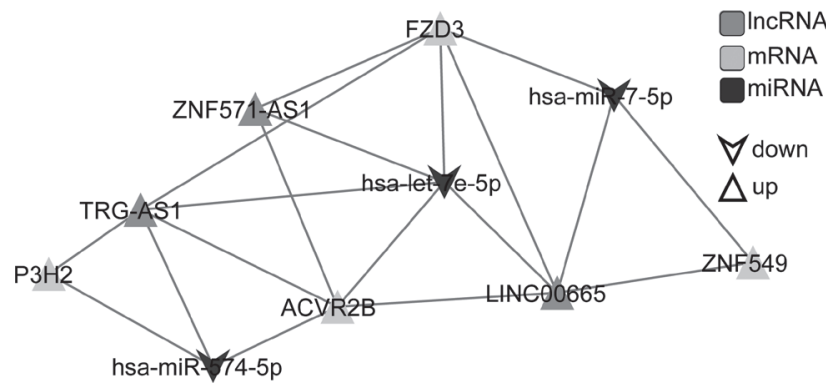

Figure 6. Construction of the ceRNA (competing endogenous RNA) network and functional analysis. Gene identification from the ceRNA network. Light gray, mRNA; dark gray, lncRNA; black, miRNA.

$\mathrm{P} 3 \mathrm{H} 2$ is involved in the 3-hydroxylation of proline in type IV collagen chains (Fernandes et al. 2011), and TRGAS1 may function as a ceRNA to inhibit the suppressive effect of hsa-miR-574-5p on $\mathrm{P} 3 \mathrm{H} 2$ during IDD progression. ZNF549 can methylate DNA and may function in cancer initiation and progression (Zhao et al. 2017). Here, we showed that the LINC00665/hsa-miR-7-5p/ZNF549 axis functions as a regulatory network in the development of IDD.

lncRNAs function as ceRNAs by sponging miRNAs, which subsequently affects the binding targets of miRNAs (Salmena et al.2011). Here, we discovered some ncRNAs that function as regulatory factors in IDD progression including LINC00665, TRG-AS1, ZNF571-AS1, hsa-miR-7-5p, hsa-let-7e-5p, and hsa-miR-574-5p. LINC0065 is a lncRNA that has been frequently reported to function as a ceRNA in various cancers, including gastric cancer, lung cancer, and hepatocellular carcinoma (Cong et al. 2019; Qi et al. 2019; Shan et al. 2019; Ji et al. 2020). Compared with LINC00665, the reports associated with lncRNA T cell receptor gamma locus antisense RNA 1 (TRG-AS1) was limited, although it functions as a ceRNA in glioblastoma, hepatocellular carcinoma and tongue squamous cell carcinoma (Xie et al. 2019; He et al. 2020; Sun et al. 2020). It was also demonstrated to be involved in regulating of repeated implantation failure $(\mathrm{Xu}$ et al. 2019). ZNF571-AS1 was only reported in one study to regulate KIT and STAT5 through the JAK/STAT signaling pathway in acute myeloid leukemia (Pan et al. 2017). In this study, we revealed the involvement of the ZNF571-AS1/let$7 e-5 p / A C V R 2 B$ and FZD3 axes in IDD regulation.

Three miRNAs, including hsa-miR-7-5p, hsa-let-7e-5p, and hsa-miR-574-5p were involved in the ceRNA network of LINC00665 and TRG-AS1. Hsa-let-7e-5p was located at the center of the ceRNA network. The let-7 miRNA family regulates cancer biology by Dicer expression and is often regarded as a collection of tumor suppressors. The hsa-let$7 \mathrm{e}-5 \mathrm{p}$ downregulation has been revealed to be related with aggressiveness and prognosis of non-small-cell lung, breast, and epithelial ovarian cancers (Mitra et al. 2011; Shi et al. 2017; Xiao et al. 2017). In esophageal squamous cell carcinoma, hsa-let-7e-5p is upregulated and acts as an oncomiR to promote the migration and invasion of cancer cells (Ma et al. 2017). Furthermore, let-7e-5p is involved in pathogenesis by regulating the expression of NLK and CASP3 and is upregulated during pre-retinal neovascularization (Desjarlais
A

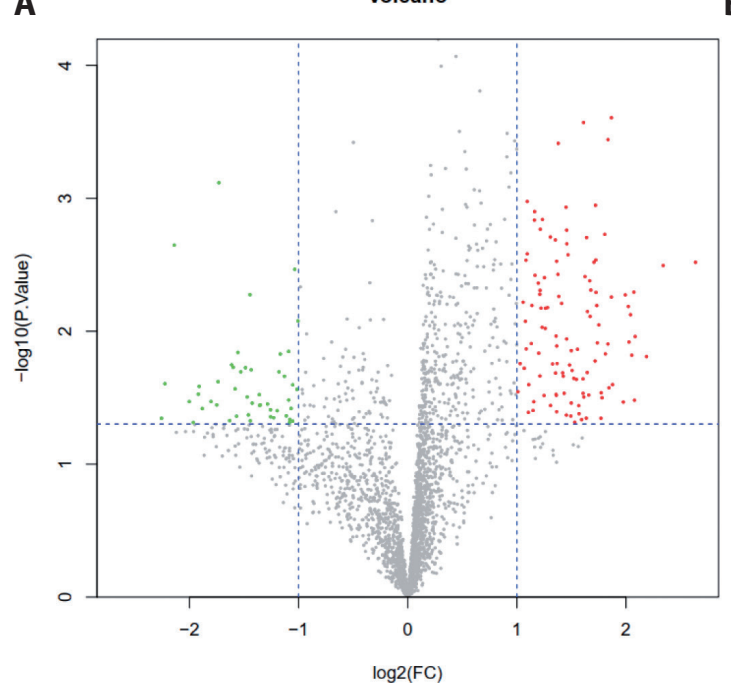

B

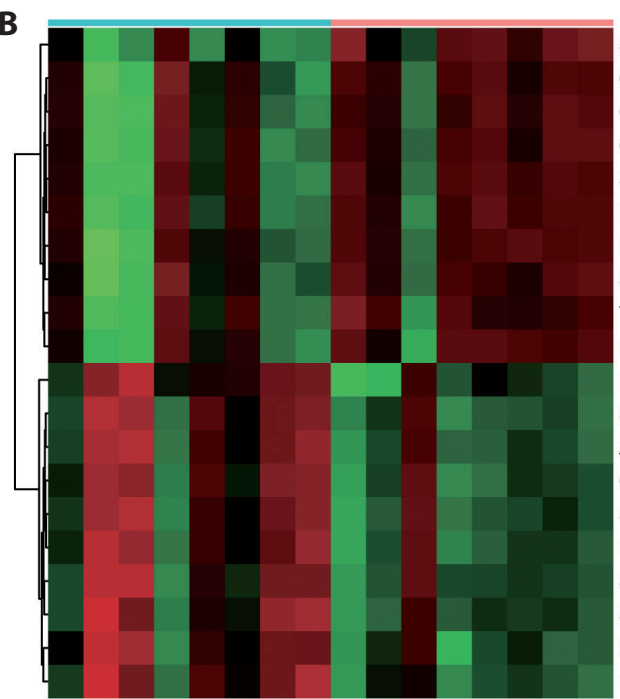

groups COL5A2:cg01247454 cg08247938 COL5A2:cg08466256 SLC4A4:cg05179645 GF1:cg17086139 BNC2:cg13471735 SLC6A17:cg07587250 TSC22D2:cg10735582 FAM184A:cg15831998 RGL3:cg24937727 SPRY1:cg13421612 ACSL1:cg27571769 OSBPL6:cg19648306 SIRPB2:cg07571344 IL1R2:cg14325025 SAMD14:cg21483922 S100B:cg00025591 PRPH:cg24453580 PTPRS:cg16902746

Figure 7. Identification of differentially methylated loci (DML) between early IDD and advanced IDD. A. Volcano plot of the DEGs. Vertical dotted lines, $|\log \mathrm{FC}| \geq 1$ and $p \leq 0.05$. Red and green spots represent upregulated and downregulated loci, respectively. B. Hierarchical clustering of the top 10 DEGs. The heatmap shows the top regulated DML in descending order according to $|\log F C| \geq 1$. For more abbreviations, see Fig. 1. 
et al. 2019). Accumulating evidence has shown that miR-7 functions in cell growth, metabolism, proliferation, apoptosis, and tumor progression (Kefas et al. 2008; Giles et al. 2016). MiR-7 inhibits hepatocellular carcinoma growth and metabolism via the PI3K/Akt pathway, targets Kruppel-like factor 4 to promote epithelial cell differentiation, inhibits colorectal cancer proliferation by regulating XRCC2, decreases cell migration and invasion, induces apoptosis by targeting focal adhesion kinase and proteasome activator subunit 3, increases the radiosensitivity of breast cancer cells via an epidermal growth factor receptor-associated signaling pathway, suppresses cell proliferation, and promotes cell cycle arrest by targeting CCNE1 (Shirota et al. 2014; Suto et al. 2014; Zhang et al. 2014; Hao et al. 2015). miR-574 has been considered as a tumor suppressor. The ectopic expression of miR-574 inhibits gastric and bladder cancer cell proliferation, invasion, and migration; suppresses cell proliferation; and induces cell apoptosis by targeting MESDC1 (Tatarano et al. 2012). To sum up, though these ncRNAs have been frequently reported in cancers, their role and regulation in IDD have not been characterized previously.

This study has some limitations. We did not obtain enough clinical samples to validate the expression of these lncRNAs, miRNAs, and mRNAs in ceRNA network. We were also unable to determine the function of methylation in IDD progression. Therefore, further investigation is still required.

\section{Conclusions}

In this study, we developed a novel ceRNA network in IDD: LICN00665/hsa-miR-7-5p/FZD3 and ZNF549, LINC00665/ hsa-let-7e-5p/FZD3 and ACVR2B, TRG-AS1/hsa-miR-5745p/ACVR2B and P3H2, TRG-AS1/ hsa-let-7e-5p/ FZD3 and ACVR2B, and ZNF571-AS1/hsa-let-7e-5p/ACVR2B and FZD3. This network serves to advance the current understanding of IDD and may be important for identifying alternative IDD therapies.

Conflict of interests. The authors declared that there is no conflict of interest.

Funding. This study was supported by the National Natural Science Foundation of China (No. 81974345).

Role of the funding source. The funding source had no involvement in study design, data collection, analysis and interpretation.

Author contributions. CYM and XTF designed the study. CYM obtained the funding. FYM and PHG acquired data. XTF, GWC, BQ and YHL analyzed and interpreted the data. XTF drafted the article and CYM revised it critically for important intellectual content. All authors read and final approved the version to be submitted.

\section{References}

Akeda K, Yamada T, Inoue N, Nishimura A, Sudo A (2015): Risk factors for lumbar intervertebral disc height narrowing: a population-based longitudinal study in the elderly. BMC Musculoskelet Disord. 16, 344 https://doi.org/10.1186/s12891-015-0798-5

Andersson GB (1999): Epidemiological features of chronic low-back pain. Lancet 354, 581-585 https://doi.org/10.1016/S0140-6736(99)01312-4

Chai G, Goffinet AM, Tissir F (2015): Celsr3 and Fzd3 in axon guidance. Int. J. Biochem. Cell Biol. 64, 11-14 https://doi.org/10.1016/j.biocel.2015.03.013

Chen J, Jia YS, Liu GZ, Sun Q, Zhang F, Ma S, Wang Y (2017): Role of LncRNA TUG1 in intervertebral disc degeneration and nucleus pulposus cells via regulating Wnt/beta-catenin signaling pathway. Biochem. Biophys. Res. Commun. 491, 668-674 https://doi.org/10.1016/j.bbrc.2017.07.146

Cong Z, Diao Y, Xu Y, Li X, Jiang Z, Shao C, Ji S, Shen Y, De W, Qiang Y (2019): Long non-coding RNA linc00665 promotes lung adenocarcinoma progression and functions as ceRNA to regulate AKR1B10-ERK signaling by sponging miR-98. Cell Death Dis. 10, 84 https://doi.org/10.1038/s41419-019-1361-3

Desjarlais M, Rivera JC, Lahaie I, Cagnone G, Wirt M, Omri S, Chemtob S (2019): MicroRNA expression profile in retina and choroid in oxygen-induced retinopathy model. PLoS One 14, e0218282

https://doi.org/10.1371/journal.pone.0218282

Fernandes RJ, Farnand AW, Traeger GR, Weis MA, Eyre DR (2011): A role for prolyl 3-hydroxylase 2 in post-translational modification of fibril-forming collagens. J. Biol. Chem. 286, 30662-30669

https://doi.org/10.1074/jbc.M111.267906

Giles K, Brown R, Ganda C, Podgorny M, Candy P, Wintle L, Richardson F, Stuart L, Epis M, Haass N, et al. (2016): MicroRNA-7-5p inhibits melanoma cell proliferation and metastasis by suppressing RelA/NF-кB. Oncotarget 7, 31663-31680 https://doi.org/10.18632/oncotarget.9421

Han HQ, Zhou X, Mitch WE, Goldberg AL (2013): Myostatin/ activin pathway antagonism: molecular basis and therapeutic potential. Int. J. Biochem. Cell Biol. 45, 2333-2347 https://doi.org/10.1016/j.biocel.2013.05.019

Hao Z, Yang J, Wang C, Li Y, Zhang Y, Dong X, Zhou L, Liu J, Zhang Y, Qian J (2015): MicroRNA-7 inhibits metastasis and invasion through targeting focal adhesion kinase in cervical cancer. Int. J. Clin. Exp. Med. 8, 480-487

He S, Wang X, Zhang J, Zhou F, Li L, Han X (2020): TRG-AS1 is a potent driver of oncogenicity of tongue squamous cell carcinoma through microRNA-543/Yes-associated protein 1 axis regulation. Cell Cycle 19, 1969-1982 https://doi.org/10.1080/15384101.2020.1786622

Hua ZL, Jeon S, Caterina MJ, Nathans J (2014): Frizzled3 is required for the development of multiple axon tracts in the mouse central nervous system. Proc. Natl. Acad. Sci. USA 111, E3005-3014 https://doi.org/10.1073/pnas.1406399111 
Ikuno A, Akeda K, Takebayashi SI, Shimaoka M, Okumura K, Sudo A (2019): Genome-wide analysis of DNA methylation profile identifies differentially methylated loci associated with human intervertebral disc degeneration. PLoS One 14, e0222188 https://doi.org/10.1371/journal.pone.0222188

Ji W, Diao YL, Qiu YR, Ge J, Cao XC, Yu Y (2020): LINC00665 promotes breast cancer progression through regulation of the miR-379-5p/LIN28B axis. Cell Death Dis. 11, 16 https://doi.org/10.1038/s41419-019-2213-x

Kefas B, Godlewski J, Comeau L, Li Y, Abounader R, Hawkinson M, Lee J, Fine H, Chiocca E, Lawler S, Purow B (2008): microRNA-7 inhibits the epidermal growth factor receptor and the Akt pathway and is down-regulated in glioblastoma. Cancer Res. 68, 3566-3572 https://doi.org/10.1158/0008-5472.CAN-07-6639

Li J, Lian Y, Yan C, Cai Z, Ding J, Ma Z, Peng P, Wang K (2017): Long non-coding RNA FOXP4-AS1 is an unfavourable prognostic factor and regulates proliferation and apoptosis in colorectal cancer. Cell Prolif. 50, e12312 https://doi.org/10.1111/cpr.12312

Li Z, Li X, Chen C, Li S, Shen J, Tse G, Chan M, Wu W (2018): Long non-coding RNAs in nucleus pulposus cell function and intervertebral disc degeneration. Cell Prolif. 51, e12483 https://doi.org/10.1111/cpr.12483

Liu X, Che L, Xie YK, Hu QJ, Ma CJ, Pei YJ, Wu ZG, Liu ZH, Fan LY, Wang HQ (2015): Noncoding RNAs in human intervertebral disc degeneration: An integrated microarray study. Genom. Data 5, 80-81 https://doi.org/10.1016/j.gdata.2015.05.027

Loreto C, Musumeci G, Castorina A, Martinez G (2011): Degenerative disc disease of herniated intervertebral discs is associated with extracellular matrix remodeling, vimentin-positive cells and cell death. Ann. Anat. 193, 156-162 https://doi.org/10.1016/j.aanat.2010.12.001

Luoma K, Riihimäki H, Luukkonen R, Raininko R, Viikari-Juntura E, Lamminen A (2000): Low back pain in relation to lumbar disc degeneration. Spine 25, 487-492 https://doi.org/10.1097/00007632-200002150-00016

Ma J, Zhan Y, Xu Z, Li Y, Luo A, Ding F, Cao X, Chen H, Liu Z (2017): ZEB1 induced miR-99b/let-7e/miR-125a cluster promotes invasion and metastasis in esophageal squamous cell carcinoma. Cancer Lett. 398, 37-45 https://doi.org/10.1016/j.canlet.2017.04.006

Mitra D, Das PM, Huynh FC, Jones FE (2011): Jumonji/ARID1 B (JARID1B) protein promotes breast tumor cell cycle progression through epigenetic repression of microRNA let-7e. J. Biol. Chem. 286, 40531-40535 https://doi.org/10.1074/jbc.M111.304865

Myouzen K, Kochi Y, Okada Y, Terao C, Suzuki A, Ikari K, Tsunoda T, Takahashi A, Kubo M, Taniguchi A, et al. (2012): Functional variants in NFKBIE and RTKN2 involved in activation of the NF-kappaB pathway are associated with rheumatoid arthritis in Japanese. PLoS Genet. 8, e1002949 https://doi.org/10.1371/journal.pgen.1002949

Pan JQ, Zhang YQ, Wang JH, Xu P, Wang W (2017): lncRNA coexpression network model for the prognostic analysis of acute myeloid leukemia. Int. J. Mol. Med. 39, 663-671 https://doi.org/10.3892/ijmm.2017.2888
Pusapati GV, Hughes CE, Dorn KV, Zhang D, Sugianto P, Aravind L, Rohatgi R (2014): EFCAB7 and IQCE regulate hedgehog signaling by tethering the EVC-EVC2 complex to the base of primary cilia. Dev. Cell 28, 483-496 https://doi.org/10.1016/j.devcel.2014.01.021

Qi H, Xiao Z, Wang Y (2019): Long non-coding RNA LINC00665 gastric cancer tumorigenesis by regulation miR-149-3p/RNF2 axis. OncoTargets Ther. 12, 6981-6990 https://doi.org/10.2147/OTT.S214588

Salmena L, Poliseno L, Tay Y, Kats L, Pandolfi PP (2011): A ceRNA hypothesis: the Rosetta Stone of a hidden RNA language? Cell 146, 353-358 https://doi.org/10.1016/j.cell.2011.07.014

Shan Y, Li P (2019): Long intergenic non-protein coding RNA 665 regulates viability, apoptosis, and autophagy via the miR-186$5 \mathrm{p} / \mathrm{MAP} 4 \mathrm{~K} 3$ axis in hepatocellular carcinoma. Yonsei Med. J. 60, $842-853$ https://doi.org/10.3349/ymj.2019.60.9.842

Shi W, Zhang Z, Yang B, Guo H, Jing L, Liu T, Luo Y, Liu H, Li Y, Gao Y (2017): Overexpression of microRNA let-7 correlates with disease progression and poor prognosis in hepatocellular carcinoma. Medicine 96, e7764 https://doi.org/10.1097/MD.0000000000007764

Shirota H, Klinman DM (2014): Recent progress concerning CpG DNA and its use as a vaccine adjuvant. Expert Rev. Vaccines 13, 299-312 https://doi.org/10.1586/14760584.2014.863715

Sun X, Qian Y, Wang X, Cao R, Zhang J, Chen W, Fang M (2020): LncRNA TRG-AS1 stimulates hepatocellular carcinoma progression by sponging miR-4500 to modulate BACH1. Cancer Cell Int. 20, 367

https://doi.org/10.1186/s12935-020-01440-3

Suto T, Yokobori T, Yajima R, Morita H, Fujii T, Yamaguchi S, Altan B, Tsutsumi S, Asao T, Kuwano H (2014): MicroRNA-7 expression in colorectal cancer is associated with poor prognosis and regulates cetuximab sensitivity via EGFR regulation. Carcinogenesis 36, 338-345 https://doi.org/10.1093/carcin/bgu242

Tatarano S, Chiyomaru T, Kawakami K, Enokida H, Yoshino H, Hidaka H, Nohata N, Yamasaki T, Gotanda T, Tachiwada T, et al. (2012): Novel oncogenic function of mesoderm development candidate 1 and its regulation by MiR-574-3p in bladder cancer cell lines. Int. J. Oncol. 40, 951-959 https://doi.org/10.3892/ijo.2011.1294

Vijayakumar S, Liu G, Rus IA, Yao S, Chen Y, Akiri G, Grumolato L, Aaronson S (2011): High-frequency canonical Wnt activation in multiple sarcoma subtypes drives proliferation through a TCF/beta-catenin target gene, CDC25A. Cancer Cell 19, 601-612 https://doi.org/10.1016/j.ccr.2011.03.010

Wang HQ, Yu XD, Liu ZH, Cheng X, Samartzis D, Jia LT, Wu SH, Huang J, Chen J, Luo ZJ (2011): Deregulated miR-155 promotes Fas-mediated apoptosis in human intervertebral disc degeneration by targeting FADD and caspase-3. J. Pathol. 225, 232-242

https://doi.org/10.1002/path.2931

Wang X, Lv G, Li J, Wang B, Zhang Q, Lu C (2017): LncRNA-RP11296A18.3/miR-138/HIF1A pathway regulates the proliferation 
ECM synthesis of human nucleus pulposus cells (HNPCs). J. Cell. Biochem. 118, 4862-4871 https://doi.org/10.1002/jcb.26166

Wang X, Zou M, Li J, Wang B, Zhang Q, Liu F, Lü G (2018): LncRNA H19 targets miR-22 to modulate $\mathrm{H} 2 \mathrm{O} 2$-induced deregulation in nucleus pulposus cell senescence, proliferation, and ECM synthesis through Wnt signaling. J. Cell. Biochem. 119, 4990-5002 https://doi.org/10.1002/jcb.26738

Wang Y, Dai G, Li L, Liu L, Jiang L, Li S, Wang F, Du W, Li Y (2019): Transcriptome signatures reveal candidate key genes in the whole blood of patients with lumbar disc prolapse. Exp. Ther. Med. 18, 4591-4602 https://doi.org/10.3892/etm.2019.8137

Xi Y, Jiang T, Wang W, Yu J, Wang Y, Wu X, He Y (2017): Long non-coding HCG18 promotes intervertebral disc degeneration by sponging miR-146a-5p and regulating TRAF6 expression. Sci. Rep. 7, 13234 https://doi.org/10.1038/s41598-017-13364-6

Xiao M, Cai J, Cai L, Jia J, Xie L, Zhu Y, Huang B, Jin D, Wang Z (2017): Let-7e sensitizes epithelial ovarian cancer to cisplatin through repressing DNA double strand break repair. J. Ovarian Res. 10, 24 https://doi.org/10.1186/s13048-017-0321-8

Xie H, Shi S, Chen Q, Chen Z (2019): LncRNA TRG-AS1 promotes glioblastoma cell proliferation by competitively binding with miR-877-5p to regulate SUZ12 expression. Pathol. Res. Pract. 215, 152476 https://doi.org/10.1016/j.prp.2019.152476

Xu H, Zhou M, Cao Y, Zhang D, Han M, Gao X, Xu B, Zhang A (2019): Genome-wide analysis of long noncoding RNAs, microRNAs, and mRNAs forming a competing endogenous RNA network in repeated implantation failure. Gene 720, 144056

https://doi.org/10.1016/j.gene.2019.144056
Yang C, Wu K, Wang S, Wei G (2018): Long non-coding RNA XIST promotes osteosarcoma progression by targeting YAP via miR195-5p. J. Cell. Biochem. 119, 5646-5656 https://doi.org/10.1002/jcb.26743

Yao RW, Wang Y, Chen LL (2019): Cellular functions of long noncoding RNAs. Nat. Cell Biol. 21, 542-551 https://doi.org/10.1038/s41556-019-0311-8

Zhang X, Hu S, Wang L, Yan B, Zhao J, Yang A, Zhang R (2014): MicroRNA-7 arrests cell cycle in G1 phase by directly targeting CCNE1 in human hepatocellular carcinoma cells. Biochem. Biophys. Res. Commun. 443, 1078-1084 https://doi.org/10.1016/j.bbrc.2013.12.095

Zhao B, Lu M, Wang D, Li H, He X (2016): Genome-wide identification of long noncoding RNAs in human intervertebral disc degeneration by RNA sequencing. BioMed Res. Int. 2016, 3684875 https://doi.org/10.1155/2016/3684875

Zhao H, Hou W, Tao J, Zhao Y, Wan G, Ma C, Xu H (2016): Upregulation of lncRNA HNF1A-AS1 promotes cell proliferation and metastasis in osteosarcoma through activation of the Wnt/beta-catenin signaling pathway. Am. J. Transl. Res. 8, 3503-3512

Zhao W, Mo Y, Wang S, Midorikawa K, Ma N, Hiraku Y, Oikawa S, Huang G, Zhang Z, Murata M, Takeuchi K (2017): Quantitation of DNA methylation in Epstein-Barr virus-associated nasopharyngeal carcinoma by bisulfite amplicon sequencing. BMC cancer 17, 489 https://doi.org/10.1186/s12885-017-3482-3

Zhu J, Zhang X, Gao W, Hu H, Wang X, Hao D (2019): lncRNA/ circRNA-miRNA-mRNA ceRNA network in lumbar intervertebral disc degeneration. Mol. Med. Rep. 20, 3160-3174 https://doi.org/10.3892/mmr.2019.10569

Received: December 7, 2020

Final version accepted: April 8, 2021 